\title{
Analysis of higher-order statistics for modulated signals
}

\author{
Shun $\mathrm{Na}$, Nan Wu, Yinghui Zhang and Yang Liu ${ }^{\mathrm{a}}$ \\ College of Electronic Information Engineering, Inner Mongolia University, Hohhot, Inner Mongolia, China
}

\begin{abstract}
The higher-order statistics method can process non-Gaussian (that needs to consider non-Gaussian, colour noise, non-liner, non-minimum phase or cycling stability message) effectively. Furthermore these methods can provide more useful message than second-order statistics based methods. In this paper, the properties of higher-order statistics are analyzed which have been widely used in communications. And the estimation of higher-order statistics of communications (amplitude modulation, frequency modulation signal, signal phase modulation signals, pulse modulation) is studied. Simulation results indicate that the methods summarized in this paper are effective.
\end{abstract}

Keywords: higher-order statistics; cumulants; moments; polyspectrum.

\section{Introduction}

Higher-order statistics have begun to find wide applicability in many fields, such as communications, sonar, radar, and so on [1-7]. Non-Gaussian signals have valuable statistics information in their highorder moments. In fact, higher-order statistics are applicable when we are dealing with non-Gaussian processes. And it has been shown that methods which exploit the non-Gaussianity of signals have some inherent advantages over second-order algorithms [6].

The concept of higher-order statistics was proposed in 1860s. From 1980s, the study of higherorder statistics in signal processing, system identification, parameter estimation, and communications was developed. In order to improve the reliability of DOA estimation in various adverse noisy environments, a new method is proposed that lies in the weighted bispectrum spatial correlation matrix in [1]. A generic frame work for the computation of the high-order statistics of the channel capacity over generalized fading channels are provided in [2]. This framework results in simple, closed-form expressions which are shown to be asymptotically tight bounds in the high signal-to-noise ratio regime of variety of fading channels. The high-order statistics are used to reduce the computational complexity and improve the sensing agility in wideband spectrum sensing scheme with compressive measurement [3]. A new method based on multiscale decomposition and high-order statistics is presented for the simultaneous solution of multiuser interference and time-varying multipath propagation in the uplink of a cellular direct-sequence spread-spectrum code-division multiple-access (DC/CDMA) system [4]. The high-order statistics were also used in direction finding and the parameters estimation of exponentially damped sinusoids signals [5], [6]. It is demonstrated the new methods are effective when the additive Gaussian noise is present. In addition, the high-order statistics are also used in automatic modulation classification for multipath fading channel [7].

\footnotetext{
${ }^{\mathrm{a}}$ Corresponding author : yangliuimu@163.com
} 
There has been an interest in applying higher-order statistics to a wide range of signal processing and system theory. The development of cumulants and polyspectra has paralleled the development of traditional correlation and its associate spectrum [8], [9]. Cumulants are blind to any kind of a Gaussian process, thus, cumulants based signal processing methods handle colored Gaussian measurement noise automatically. Furthermore, polyspectra defined in terms of higher-order cumulants of the process contain information regarding deviations from Gaussianness and presence of nonlinearities. In this paper, we address the problem of analysis of higher-order statistics for some modulated signals in the presence of Gaussian noise. The definition of cumulants and polyspectra are described. The higher-order statistics of several useful modulated signals are analyzed and simulated.

\section{Definitions and properties of higher-order statistics}

Let $x$ are random variables, the $k$ th -order central moments is defined by

$$
\mu_{k}=E\left[(x-\eta)^{k}\right]=\int_{-\infty}^{\infty}(x-\eta)^{k} p(x) d x,
$$

where $\eta$ is the average value of $x$. It follows from (1) that $\mu_{0}=1, \mu_{1}=0$, and $\mu_{2}=\sigma^{2}$. If $x$ is a zero mean process, then

$$
m_{k}=E\left[x^{k}\right]=\int_{-\infty}^{\infty} x^{k} p(x) d x
$$

which is called ordinary moments. If the ordinary moments $m_{k}$ is exist, the characteristic function of $x$ can be represented in Taylor series

$$
\Phi(\omega)=1+\sum_{k=1}^{n} \frac{m_{k}}{k !}(j \omega)^{k}+O\left(\omega^{n}\right)
$$

The second characteristic function is defined by

$$
\Psi(\omega)=\ln \Phi(\omega)=\sum_{k=1}^{n} \frac{c_{k}}{k !}(j \omega)^{k}+O\left(\omega^{n}\right)
$$

where $c_{k}$ is denote to the $k$ th cumulants,

$$
c_{k}=\left.\frac{1}{j^{k}}\left[\frac{d^{k}}{d \omega^{k}} \ln \Phi(\omega)\right]\right|_{\omega=0}=\left.\frac{1}{j^{k}}\left[\frac{d^{k} \Psi(\omega)}{d \omega^{k}}\right]\right|_{\omega=0}=(-j)^{k} \Psi^{k}(0), \quad k \leq n .
$$

The moments and cumulants can be related to one another. Let $n \rightarrow \infty$, the characteristic function is represent by

$$
\begin{aligned}
\Phi(\omega) & =1+\sum_{k=1}^{\infty} \frac{m_{k}}{k !}(j \omega)^{k}=\exp \left[\sum_{k=1}^{\infty} \frac{c_{k}}{k !}(j \omega)^{k}\right] \\
& =1+\sum_{k=1}^{\infty} \frac{c_{k}}{k !}(j \omega)^{k}+\frac{1}{2 !}\left[\sum_{k=1}^{\infty} \frac{c_{k}}{k !}(j \omega)^{k}\right]^{2}+\mathrm{L}+\frac{1}{n !}\left[\sum_{k=1}^{\infty} \frac{c_{k}}{k !}(j \omega)^{k}\right]^{n}+\mathrm{L} .
\end{aligned}
$$

Compare the parameters of $(j \omega)^{k}(k=1,2, \mathrm{~L})$, the relation between moments and cumulants is given by,

$$
c_{1}=m_{1}
$$




$$
\begin{aligned}
& c_{2}=m_{2}-m_{1}^{2}=E\left[x^{2}\right]-(E[x])^{2}=E\left[(x-E[x])^{2}\right]=\mu_{2} \\
c_{3}= & m_{3}-3 m_{1} m_{2}+2 m_{1}^{3} \\
= & E\left[x^{3}\right]-3 E[x] E\left[\left(x^{2}\right)\right]+2(E[x])^{3}=E\left[(x-E[x])^{3}\right]=\mu_{3} \\
c_{4}= & m_{4}-3 m_{2}^{2}-4 m_{1} m_{3}+12 m_{1}^{2} m_{2}-6 m_{1}^{4} \neq E\left[(x-E[x])^{4}\right]=\mu_{4} .
\end{aligned}
$$

For a zero-mean stationary random process $x(t)$, the $k$ th -order cumulant of this process is defined as the joint $k$ th -order cumulant of the random variables $x(t), x\left(t+\tau_{1}\right), \cdots, x\left(t+\tau_{k-1}\right)$, i.e.,

$$
c_{k, x}\left(\tau_{1}, \tau_{2}, \cdots, \tau_{k-1}\right)=\operatorname{cum}\left(x(t), x\left(t+\tau_{1}\right), \cdots, x\left(t+\tau_{k-1}\right)\right)
$$

The second-order, third-order and fourth-order cumulants of zero-mean $x(n)$ are

$$
\begin{aligned}
& c_{2, x}(m)=E[x(n) x(n+m)] \\
& c_{3, x}\left(m_{1}, m_{2}\right)=E\left[x(n) x\left(n+m_{1}\right) x\left(n+m_{2}\right)\right] \\
& c_{4, x}\left(m_{1}, m_{2}, m_{3}\right)=E\left[x(n) x\left(n+m_{1}\right) x\left(n+m_{2}\right) x\left(n+m_{3}\right)\right] \\
& -c_{2, x}\left(m_{1}\right) c_{2, x}\left(m_{2}-m_{3}\right) \\
& -c_{2, x}\left(m_{2}\right) c_{2, x}\left(m_{3}-m_{1}\right) \\
& -c_{2, x}\left(m_{3}\right) c_{2, x}\left(m_{1}-m_{2}\right)
\end{aligned}
$$

It is obvious that if $x(n)$ is Gaussian then the cumulants are all zero, except for $k=3$ and 4 . Thus, Cumulants not only display the amount of higher-order correlation, but provide a measure of the distance of the random process form Gaussianity [8]

Let $x(t)$ is zero-mean stationary process, the $k$ th -order moment of it is $m_{k, x}\left(\omega_{1}, \omega_{2}, \ldots, \omega_{n}\right)$. If $k$ th -order moment is absolutely summable,

$$
\sum_{\omega_{1}=-\infty}^{\infty} \ldots \sum_{\omega_{k-1}=-\infty}^{\infty}\left|m_{k, x}\left(\omega_{1}, \omega_{2}, \ldots \omega_{k-1}\right)\right|<\infty .
$$

The $k$ th -order moment spectra is defined as

$$
M_{k, \omega}\left(\omega_{1}, \omega_{2}, \ldots \omega_{n}\right)=\sum_{\omega_{1}=-\infty}^{\infty} \ldots \sum_{\omega_{k-1}}^{\infty} m_{k, \omega}\left(\omega_{1}, \omega_{2}, \ldots \omega_{k-1}\right) \exp \left[-j \sum_{i=1}^{k-1} \omega_{i} k_{i}\right] .
$$

There are some important properties of cumulants, which are used in theoretical analysis [8]. If $\lambda_{i}$, $i=1, \cdots, k$, are constant, and $x_{i}, i=1, \cdots, k$, are random variables, then

$$
\operatorname{cum}\left(\lambda_{1} x_{1}, \mathrm{~L}, \lambda_{k} x_{k}\right)=\prod_{i=1}^{k} \lambda_{i} \operatorname{cum}\left(x_{1}, \mathrm{~L}, x_{k}\right) .
$$

Cumulants are symmetric in their arguments,

$$
\operatorname{cum}\left(x_{1}, \mathrm{~L}, x_{k}\right)=\operatorname{cum}\left(x_{i_{1}}, x_{i_{2}}, \mathrm{~L}, x_{i_{k}}\right) \text {. }
$$

Cumulants are additive in their arguments,

$$
\operatorname{cum}\left(x_{0}+y_{0}, z_{1}, \mathrm{~L}, z_{k}\right)=\operatorname{cum}\left(x_{0}, z_{1}, \mathrm{~L}, z_{k}\right)+\operatorname{cum}\left(y_{0}, z_{1}, \mathrm{~L}, z_{k}\right)
$$


If the random variables $\left\{x_{i}\right\}$ are independent of the random variables $\left\{y_{i}\right\}, i=1, \cdots, k$, then

$$
\operatorname{cum}\left(x_{1}+y_{1}, \mathrm{~L}, x_{k}+y_{k}\right)=\operatorname{cum}\left(x_{1}, \mathrm{~L}, x_{k}\right)+\operatorname{cum}\left(y_{1}, \mathrm{~L}, y_{k}\right) \text {. }
$$

Assuming that $c_{k, x}\left(\tau_{1}, \tau_{2}, \cdots, \tau_{k-1}\right)$ is absolutely summable

$$
\sum_{m_{1}=-\infty}^{\infty} \mathrm{L} \sum_{m_{k-1}=-\infty}^{\infty}\left|c_{k, x}\left(m_{1}, m_{2}, \mathrm{~L}, m_{k-1}\right)\right|<\infty
$$

the $k$ th -order polyspectrum is defined as the $k-1$ dimensional Fourier transform of the $k$ th -order cumulant,

$$
S_{k, x}\left(\omega_{1}, \omega_{2}, \mathrm{~L}, \omega_{k-1}\right)=\sum_{m_{1}=-\infty}^{\infty} \mathrm{L} \sum_{m_{k-1}=-\infty}^{\infty} c_{k, x}\left(m_{1}, m_{2}, \mathrm{~L}, m_{k-1}\right) \exp \left[-j \sum_{i=1}^{k-1} \omega_{i} m_{i}\right]
$$

In general, the $k$ th -order polyspectrum is related to the $k-1$-order spectrum, i.e. $S_{2, x}(\omega)$ is known as the power spectrum, $S_{3, x}\left(\omega_{1}, \omega_{2}\right)$ is known as the bispectrum $\left(B_{x}\left(\omega_{1}, \omega_{2}\right)\right), S_{4, x}\left(\omega_{1}, \omega_{2}, \omega_{3}\right)$ is known as the trispectrum. The bispectrum is period function with period $2 \pi$

$$
B\left(\omega_{1}, \omega_{2}\right)=B\left(\omega_{1}+2 \pi, \omega_{2}+2 \pi\right) .
$$

And it is symmetric

$$
\begin{aligned}
B\left(\omega_{1}, \omega_{2}\right) & =B\left(\omega_{2}, \omega_{1}\right) \\
& =B^{*}\left(-\omega_{2},-\omega_{1}\right)=B^{*}\left(-\omega_{1},-\omega_{2}\right) \\
& =B\left(-\omega_{1}-\omega_{2}, \omega_{2}\right)=B\left(\omega_{1},-\omega_{1}-\omega_{2}\right) \\
& =B\left(-\omega_{1}-\omega_{2}, \omega_{1}\right)=B\left(\omega_{2},-\omega_{1}-\omega_{2}\right) .
\end{aligned}
$$

These properties make bispectrum become to a useful technique for signal processing [9].

\section{Higher-order statistics of modulated signals}

Depending on the nature of the communication channel, signal can suffer from one or more of certain channel impairment including noise, attenuation, distortion, fading, and interference. To transmit the signal over the channel, it needs to generate a modulated signal that represents the source information and matches the characteristics of the channel. Science different channels cause different types of impairments, modulations designed for these channels can be drastically different [10]. In communications systems, analog modulation and digital modulation are the most commonly used modulation schemes. In this chapter, we simulate the higher-order statistics of amplitude modulation $(\mathrm{AM})$, frequency modulation (FM), phase modulation (PM), and quadrature amplitude modulation (QAM) signals.

Amplitude Modulation (AM) is the amplitude of the carrier wave (high frequency) signal controls the instantaneous amplitude modulated signal by. That is to say, that the frequency of the carrier signal according to a modulation do periodic variation, strength against their amplitude of the modulation signal is linear but the carrier frequency and phase remain constant, independent of the modulation signal, the amplitude of the high frequency oscillation change the response signal carrying information. The high-frequency signals through antennas transmit out, but put the modulation signal is also spread out. This time at the receiving end can be demodulated modulation signal is demodulated method, that is, the amplitude of the high frequency carrier signal is deciphered the modulated signal can be obtained. The fourth-order cumulants of a double-side AM signal with carrier frequency of $f=0.25 f_{s}$ and bandwidth of $B=0.1875 f_{s}$ without noise and in the presence of 
Gaussian noise are shown in Figure 1. The signal to noise ratio (SNR) is OdB. Since the source data is Gaussian, it can be seen from Figure 1 that the fourth-order cumulants are nearly equals to zero.

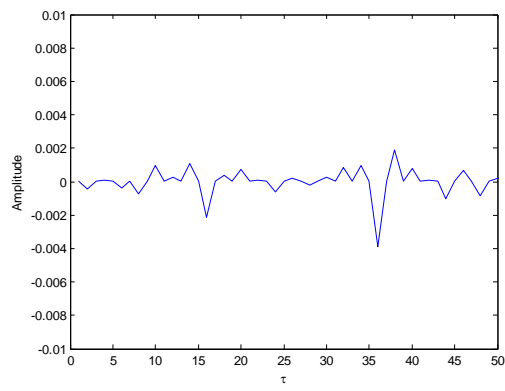

(a)

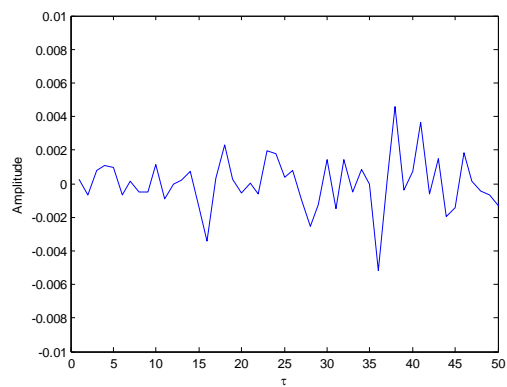

(b)

Figure 1. Fourth-order cumulants of AM signal. (a) Fourth-order cumulants of the signal only. (b) Fourth-order cumulants in Gaussian noise

For the frequency modulation (FM), the instantaneous frequency of the carrier is changed as the strength of the source. But the amplitude of the carrier is not affected by the source data. Its amplitude still retains its original size. However, the bandwidth of FM required to be much larger than that of the AM modulation. The information of FM signals is contained in the frequency changing, and general interference the main causes changes in signal amplitude, thus it is easy to eliminate the $f$ amplitude variation of the interference by the limiter. Under normal circumstances, interfering signals always superimposed on the amplitude of source signal. Therefore, FM wave can avoid the influence of noise amplitude interference and thus improve the quality of communication. FM modulation is widely used in communications, radio and television. The third-order and fourth-order cumulants of a FM signal is shown in Figure 2. The bispectrum of the FM signal is shown in Figure 3.

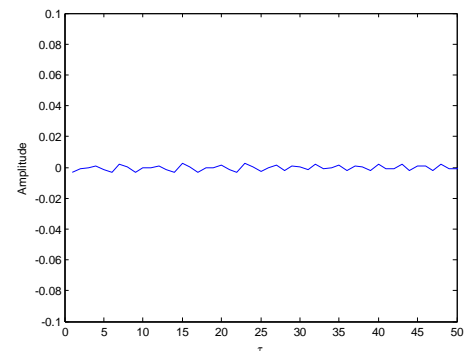

(a)

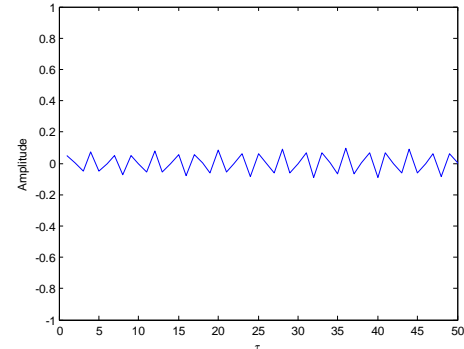

(b)

Figure 2. Third-order and fourth-order cumulants of FM signal. (a) Third-order cumulant. (b) Fourth-order cumulant

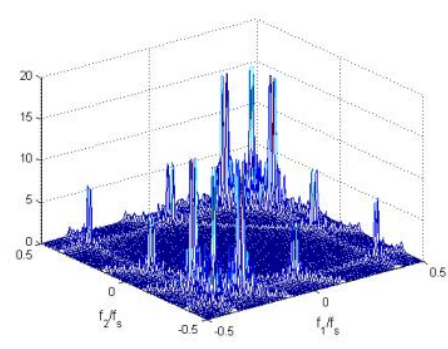

(a)

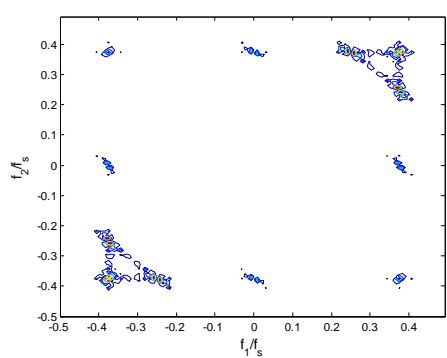

(b)

Figure 3. Bispectrum of the FM signal. (a) The bispectrum. (b) Contour of bispectrum 


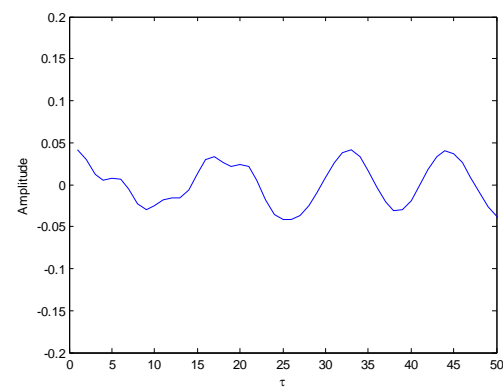

(a)

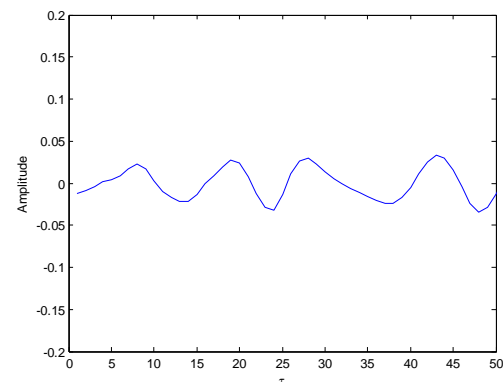

(b)

Figure 4. Third-order and fourth-order cumulants of FM signal. (a) Third-order cumulant. (b) Fourth-order cumulant

In phase modulation (PM), the instantaneous phase of the carrier (high frequency) is a linear relationship between the change in period is determined by the frequency modulation signal. However, the amplitude of the modulated signal remains unchanged without being affected by the modulation signal. PM also has a strong anti-jamming capability, the general is mainly used in digital communication systems phase shift keying. Compared with the amplitude modulation, the most important advantage of frequency modulation and phase modulation is the strong interference carve. The third-order and fourth-order cumulants of a PM signal is shown in Figure 4. Figure 5 shows the bispectrum of the PM signal

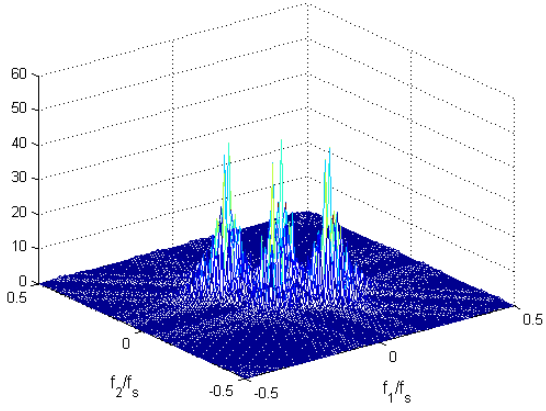

(a)

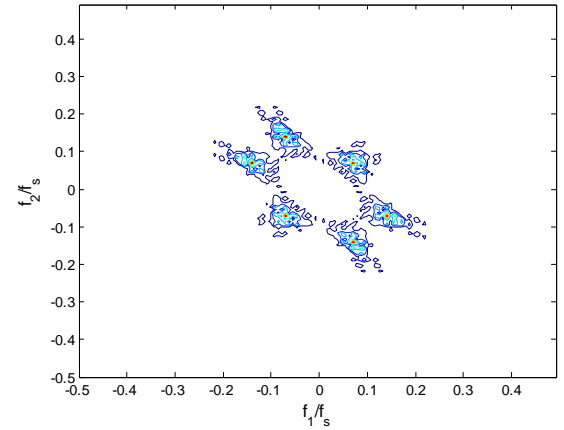

(b)

Figure 5. Bispectrum of the PM signal. (a) The bispectrum. (b) Contour of bispectrum

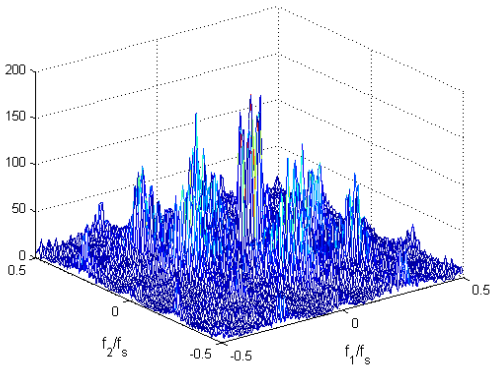

(a)

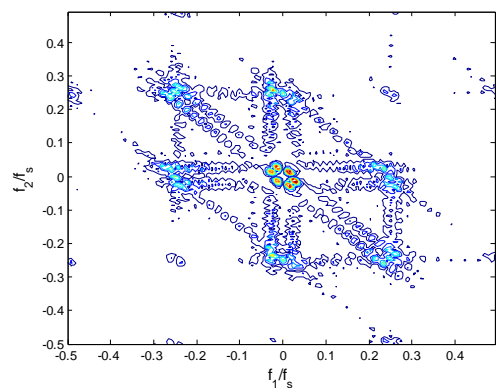

(b)

Figure 6. Bispectrum of the QAM signal. (a) The bispectrum. (b) Contour of bispectrum.

The process of mapping a digital sequence to signals for transmission over a communications channel is called digital modulation. The general digital modulation schemes are linear modulation schemes and nonlinear modulation schemes. In QAM signaling the lowpass equivalent of the signal is 
of the form $A_{m} g(t)$ where $A_{m}$ is a complex number. Therefore the sum of two lowpass equivalent signals corresponding to two different signals is of the general form of the lowpass equivalent of a 32QAM signal. The bispectrum of the QAM signal is shown in Figure 6.

\section{Conclusions}

In this paper, we have focused on higher-order statistics of modulated signals. In the presence of nonGaussianity, color noise, and nonlinearities, higher-order statistics offer an effective set of methodologies and algorithms for handling these effects. In addition, various areas of application that can directly benefit from the use of the high-order statistics. And it turns out to be useful in the case of non-Gaussian process with zero measure of skewness. Furthermore, polyspectrum is useful for filtering impulsive noise and time delay estimation.

\section{Acknowledgement}

This work is supported by National Science Foundation of China, Nature Science Foundation of Inner Mongolia of China. Work partially supported by grant61362027, 61461036 of the China National Science Foundation, and 2016MS0604 of the Nature Science Foundation of Inner Mongolia of China .

\section{References}

1. Wei Xue, Wenjun Liu, Shan Liang, Noise robust direction of arrival estimation for speech source with weighted bispectrum spatial correlation matrix, IEEE Transactions on Signal Processing837-851, 9(2015)

2. F. Yilmaz, M. S. Alouini, Novel asymptotic results on the high-order statistics of the channel capacity over generalized fading channels, in: IEEE 13th international workshop on signal processing advances in wireless communications, (2012).

3. Kaitian Cao, Hequn Shao, Compressive wideband spectrum sensing using high-order statistics for cognitive radio, in: 2013 IEEE Global high tech congress on electronics, (2013).

4. M. Martone, Blind adaptive detection of DS/CDMA signals on time-varying multipath channels with antenna arrays using high-order statistics, IEEE Transactions on Communications 1590-1600, 9(2000).

5. B. Porat, B. Friedlander, Direction finding algorithms based on high-order statistics, IEEE Transactions on Signal Processing2016-2024, 9(1991).

6. C. K. Papadopoulos, C. L. Nikias, Parameter estimation of exponential damped sinusoids using high order statistics, IEEE Transactions on Signal Processing1424-1436, 9 (1991).

7. P. Shih, D. C. Chang, An automatic modulation classification technique using high-order statistics for multipath fading channels, in: 2011 11th international conference on ITS telecommunications, (2011).

8. J. M. Mendel, Tutorial on higher-order statistics (spectra) in signal processing and system theory: theoretical results and some applications, Proceedings of the IEEE278-305, 3 (1991) .

9. C. L. Nikias, M. R. Raghuveer,Bispectrum estimation: a digital signal processing framework, Proceedings of the IEEE869-891, (1987).

10. J. G. Proakis, Digital Communications,5th edn.(McGraw-Hill,2009). 